\section{Rare diseases in the elderly: a new perspective for the specialist in geriatrics}

Carlo Sabbà, Gennaro M. Lenato, Carlo Custodero, Patrizia Suppressa

Frugoni Internal Medicine and

Geriatric Unit, Center for Rare

Diseases, Interdisciplinary Department

of Medicine, Interdepartmental HHT

Center and VASCERN HHT European

Reference Centre, University of Bari

School of Medicine, Policlinico Hospital,

Bari, Italy

\section{Abstract}

Rare diseases (RD) encompass a broad spectrum of highly heterogeneous illnesses characterized by a prevalence lower than 1:2000 in general population. Although relatively uncommon, when considered as a whole, these pathologies represent a relevant public health problem.

In light of their usual early onset and high severity and mortality, RD have been traditionally regarded as affecting mainly childhood or young adulthood, and considered to be excluded from filed of interest of geriatric specialists.

However, more recent epidemiological studies, suggest that demographic changes, sometimes joined with advances in diagnostic and therapeutic strategies, are permitting longer survival of some persons affected by these conditions, thus making RD compatible with geriatric age.

Hence, in the next future, specialists in geriatrics will be referred to by an increasing number of elderly subjects with RD seeking medical care. Geriatricians should be aware about the high management complexity of elderly patients with RD, characterized by considerable frailty status, related to both the primary $\mathrm{RD}$ and to the ageingrelated condition.

\section{Introduction}

Rare diseases (RD) represent a collective term, including numerous and highly heterogeneous illnesses, which share the peculiarity to affect a limited number of individuals in the population. ${ }^{1}$ In particular, the definition of RD in European Union applies for diseases with a prevalence lower than 1:2000 subjects, which accounts for an estimated number of distinct RD between
5000 and $8000 .^{2}$ It is important to emphasize that $\mathrm{RD}$ do not constitute a defined nosological grouping, as these conditions considerably differ in terms of etiology, clinical features, severity, onset age. Despite such large inhomogeneity, attention to these disorders was focused in the 1970s of the last century and the concept of rare diseases was firstly evidenced, ${ }^{3,4}$ based on the consideration that subjects living with $\mathrm{RD}$ usually experience a range of common problems, directly linked to the rarity of these pathologies, including difficult diagnosis, scarce availability of therapeutic options, lack of attention by medical specialists, sense of isolation (Table 1). ${ }^{2}$

Until recently, low awareness has been devoted to the aspects related to RD by a large part of the scientific community and policy makers. ${ }^{5}$ Clinical trials, research on pathogenic mechanisms, and epidemiological descriptions are hampered by the very low numbers of cases and their dispersion, as well as by difficulties in amortizing the costs of pharmacological studies. ${ }^{6}$ Such scarcity of information often results in disparity in terms of access to care, standardization of healthcare pathways, social support allocations. For these reasons, these pathologies are referred to as orphan diseases. ${ }^{1,2,7}$

In recent years, however, increasing epidemiological and scientific data pointed out that, despite the rarity of the single disease, when RD are considered collectively, they affect a remarkable portion of the population (at least 13.5 million in European Union), ${ }^{2}$ thus becoming a paramount public and social healthcare issue. ${ }^{1,5}$

\section{The pathway of patients with rare disease}

Patients and families with a rare disease are usually involved in a complex, and often lifelong, healthcare pathway, coming into contact with various types of professional healthcare providers. ${ }^{8}$ The paucity of information available in $\mathrm{RD}$ impacts at different levels on the healthcare pathway of the affected individual, as well as on family members and caregivers (diagnosis, monitoring, support, therapy, follow-up). Subjects with RD and their families face several hurdles, such as delayed diagnosis, geographical scattering of expertise centers and/or trained professionals, lack of effective therapeutic options, chronic disability trajectories, familial inheritability. $2,6,9$

Throughout the pathway, the first major barrier experienced by most RD-affected individuals is represented by the diagnostic delay. ${ }^{9-11}$ Several studies have shown that,
Correspondence: Carlo Sabbà, Frugoni

Internal Medicine and Geriatric Unit, Interdisciplinary Department of Medicine, Policlinico, P.zza Giulio Cesare 11, 70124 Bari, Italy.

Tel.: +39.080 .5594037$

Fax: +39.080 .5478708$

E-mail: carlo.sabba@uniba.it

Key words: Rare diseases; diagnostic delay; elderly; quality of life; hereditary hemorrhagic telangiectasia; frailty.

Contributions: the authors contributed equally.

Conflict of interests: the authors declare no potential conflict of interests.

Received for publication: 16 December 2019 Accepted for publication: 18 December 2019.

This work is licensed under a Creative Commons Attribution-NonCommercial 4.0 International License (CC BY-NC 4.0).

${ }^{\circ}$ Copyright: the Author(s), 2019

Licensee PAGEPress, Italy

Geriatric Care 2019; 5:8769

doi:10.4081/gc.2019.8769

for many $\mathrm{RD}$, the attainment of a correct diagnosis often entails a delay of many months or even years. Studies performed in hereditary hemorrhagic telangiectasia (HHT), familial mediterranean Fever (FMF), and Ehlers-Danlos syndrome (EDS), reported an average diagnostic delay of 15-25 years. ${ }^{9,10}$ A number of factors, including the lack of availability of adequate diagnostic tools, the multidisciplinary nature of the disorder, the prominent phenotypic heterogeneity, and the variability of presentation symptoms, may be evoked to account for an objective difficulty of timely diagnosis by both generalist or specialist clinicians. ${ }^{12}$ More in details, when challenged by an undiagnosed RD patient, clinicians are often driven by textbook prototypical cases, as the rarity of the disease itself will prevent them from establishing a robust knowledge by direct experience. Such phenomenon will induce relevant cognitive bias in the diagnostic process of a RD, which can be synthesized in the general principle We recognize what we already know, historically attributed to Socrates (Vth Century B.C.). ${ }^{10}$

Once the correct diagnosis is reached, patients acquire awareness on being affected by a condition lacking medical treatment effective to clear up their disease, requiring multi-disciplinary approach, and characterized by the multiple issues, as described in previous paragraph. ${ }^{1,2}$ Hence, several studies have reported a significant reduction in 
quality of life in persons with RD., ${ }^{9}$ The considerable burden exerted by RD on the lives of affected persons often results in a sense of social and psychological isolation. ${ }^{9,11}$ Finally, most RD exert a marked effect on survival. ${ }^{8}$ A recent populationbased investigation reported that $\mathrm{RD}$, when globally considered, accounted for $4.2 \%$ of the total years of life lost (YLLs) by the general population. ${ }^{14}$ Noteworthy, mortality rates differ substantially across different $\mathrm{RD}$, since some pathologies are associated to very early fatality events ( $<1$ yr.-old), whereas other disorders show a longer survival, with diverse severity degrees.

Due to the aforementioned reasons, the presence of a rare disease determines a state of multidimensional vulnerability, which can be observed from different viewpoints (biological, clinical, social, psychological). These vulnerability factors are expected to contribute to the progressive deterioration in functioning across multiple physiological systems, thus triggering a condition of frailty, typical of RD patients. ${ }^{1,15}$ This trajectory towards frailty state is likely to represent a general features of RD, in spite of their clinical and pathophysiological heterogeneity.

\section{Rare diseases in the elderly}

For long time, RD have been traditionally regarded as being pertinent to the specialist in paediatrics, ${ }^{2,14}$ rather than in geriatrics. ${ }^{6}$ Such view is mainly based on the consideration that the vast majority of these disorders have a genetic etiology and a substantial proportion of them, such as most of complex congenital malformations, or several inborn errors of metabolism, are actually associated to very early onset (neonatal/infantile) and high mortality rates, resulting in a very short lifespan. On the other hand, it is of note that more than one third of RD have an onset in adulthood and that a growing number of pediatric patients with previously fatal RD now survive into adulthood, owing to early diagnosis and improvement of care options. ${ }^{14}$ However, based on the severity of the manifestations and the marked impact on life expectancy of these disorders, the traditional viewpoint has long assumed RD as not being compatible with reaching geriatric age.

More profound observations of the RDrelated epidemiological profile suggest a quite different scenario, especially when analyzed in the context of the phenomenon of the demographic transition displayed by the high-income nations. Indeed, in countries characterized by the well-known global aging of the population, a non-negligible number of patients suffering from RD reach the geriatric age. In a preliminary survey carried out in Italian Apulia Region, on a sample of 69,902 Hospital Discharge Records, 927 patients aged $>65$ yrs. were found to be affected by a RD. ${ }^{15}$ An epidemiological survey, based on Regional Registry of Rare Diseases in the population of Italian Veneto Region, reported an overall prevalence of 1.7 per thousand RD-affected subjects in the $>65$-yrs-old class, compared to an average value of 3.3 per thousand in the entire population, thus indicating a fraction of $9.1 \%$ of RD-affected subjects exceeding the threshold of geriatric age. ${ }^{16}$

Such observation suggests that the generalized trend towards population aging, combined with specific advances in screening and treatment for some RD, will lead the specialist in geriatrics to face the challenge of managing elderly subjects with a RD.

A paradigmatic example is HHT, a rare autosomal-dominantly-inherited vascular dysplasia, showing a broadly heterogeneous clinical spectrum, in terms of onset age, clinical course, severity of manifestations. ${ }^{17}$ The hallmark of HHT is the presence of multi-systemic arterio-venous malformations, abnormal direct connections between arterial and venous vascular bed, bypassing capillary network, responsible of number of symptoms/complications, including epistaxis, stroke, brain abscess, hypoxemia, gastrointestinal bleeding, high-output heart failure, portal hypertension. ${ }^{18,19}$ HHT may remain silent/oligosymptomatic for decades and then manifest with sudden life-threatening complications. ${ }^{10}$ Life expectancy in HHT can be compatible with advanced age, showing on average a modest survival reduction, featuring two evident mortality peaks (an early peak, due to brain/lung complications, and a late peak at older ages, due to nosebleeds worsening). ${ }^{20}$ As the proportion of HHT-affected individuals achieving the geriatric age threshold is currently growing, the specialist in geriatrics is expected to take in charge an increasing number of these subjects, who will come to his observation, presumably with different presentations. For example, some HHT patients can now benefit from appropriate preventive screening survey and avoid potentially fatal brain/lung complications, thus reaching geriatric age, while others display early-onset progressively uncontrollable nosebleeds and associated chronic debilitating iron-deficiency anemia, markedly affecting quality of life but still compatible with advanced age. ${ }^{13,21}$ Another subset of patients remains completely asymptomatic, thus experiencing disease onset only after 65 yrs.-old, and a last subgroup is characterized by a long diagnostic delay, with onset as young or middle-aged adults and attainment of correct diagnosis after geriatric age threshold. ${ }^{10,18}$ A similar scenario is expected to occur for other RD, such as haemophilia ${ }^{22,23}$ or Charcot-MarieTooth type $1 .{ }^{24}$

\section{Frailty in the elderly with rare disease}

The specialist in geriatrics is very familiar with the concept of frailty. Frailty is characterized by a functional decline across multiple physiological systems, due to reduced homeostatic capacities, which usu-

Table 1. Characteristics and issues shared by rare diseases.

\author{
Insufficient knowledge among medical/healthcare professionals \\ Diagnostic challenges, cognitive bias in diagnostic process (we recognized what we already know) \\ Lack of interest and funding for research projects \\ Scarce availability of etiologic/effective therapeutic options \\ Limited statistical power in clinical trials \\ Severe debilitating condition, difficulty in everyday management, loss of social opportunities \\ Sense of isolation, due to the rarity of the condition \\ Multi-systemic involvement, need for multi-specialist approach to care \\ Heavily compromised quality of life
}


ally predisposes to increased risk of adverse clinical outcomes. ${ }^{25}$

Given the growing relevance of RD also in the geriatric population, the frailty assessment will acquire a new perspective in elderly subjects with RD. It is plausible that the frailty associated with the condition of $\mathrm{RD}$ should be regarded in the context of the frailty status typical of the elderly. ${ }^{15}$ Henceforth, frailty in an elderly with RD will derive from a complex interaction of agingrelated vulnerability with the RD-associated vulnerability which can occur at all levels (biological, clinical, psychological, social). As shown in Figure $1,{ }^{26}$ in seniors with RD, the trend of the frailty profile is expected to display a sharper increase in relation to age, when compared with the basal situation of the general elderly population, thus mirroring the disease-related augmented disruption of functional capacity.

\section{Healthcare complexity in the elderly with rare disease}

The presence of advanced age in an individual with RD adds considerable com- plexity in the healthcare management of these pathologies. For many RD, the phenotypic spectrum in the elderly still lacks reliable and full description, in that achieving the geriatric threshold was not compatible with these disorders until very recently. Furthermore, the clinical picture and the course of the RD are inserted in the typical pluri-pathology scenario of the geriatric age, thus displaying multiple concomitant diseases, which can arise as comorbidities (representing additional clinical manifestations, stemming from the RD, potentially worsening with age), or multimorbidities (representing long-term, aging-associated pathological entities, co-occurring in the same individual and apparently independent from the RD). ${ }^{15,22}$

Complexity of interactions between the primary rare disorder with co-morbidities and/or multi-morbidities can impact on healthcare at multiple levels. ${ }^{15}$ The reported difficulties concerning diagnostic delay in $\mathrm{RD}$ are even more evident in the elderly with RD, since the concomitance of comorbidities and multimorbidities may obscure RD-related manifestations and complicate the diagnostic process. ${ }^{3}$ The lack of effective therapeutic protocols afflicting $\mathrm{RD}$ is

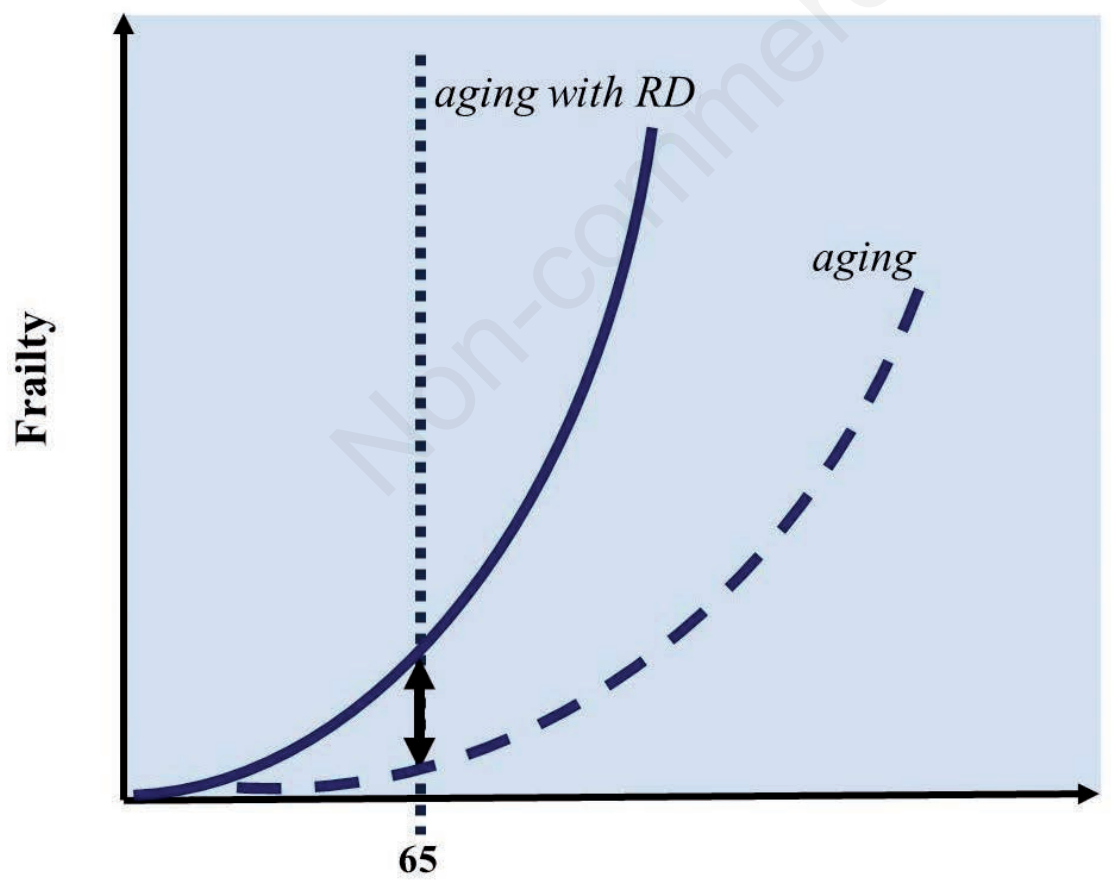

Age (years)

Figure 1. Increase of frailty in the elderly with rare disease (RD). When a person with RD enters geriatric age, vulnerability related to the rare primary condition interacts with frailty status associated to advanced age, leading to a sharper increase of frailty curve. (adapted from Sabbà C, Lenato GM, Suppressa P. Malattie "rare" in tarda età. In: Antonelli Incalzi $R$, Cesari M, Ungar A, Pedone C, Volpato S, Leosco D. Manuale di Geriatria. Milano, EDRA S.p.a. editions; 2019. pp. 495-507)

exacerbated in advanced age, due to ageingrelated pharmacokinetics and pharmacodynamics alterations, or to polypharmacyrelated issues. ${ }^{22,27}$

\section{Conclusions}

In conclusion, the dramatic demographic changes that are occurring in the third millennium will permit to many $\mathrm{RD}$ to become compatible with advanced age. Thus, an increasing number of elderly patients with RD will seek medical care from geriatric specialists in the next future.

The challenge of the geriatrician of the III millennium will therefore consist in recognizing and managing these clinical entities, with associated comorbidities and multimorbidities, according to a global, holistic, and patient-centered approach.

\section{References}

1. Schieppati A, Henter JI, Daina E, Aperia A. Why rare diseases are an important medical and social issue. Lancet 2008;371:2039-41.

2. Ayme S, Schmidtke J. Networking for rare diseases: a necessity for Europe. Bundesgesundheitsblatt Gesundheitsforschung Gesundheitsschutz 2007;50: 1477-83.

3. Holtzman NA. Rare diseases, common problems: recognition and management. Pediatrics 1978;62:1056-60.

4. Raine DN. The need for a national policy for the management of inherited metabolic disease. J Clin Pathol Suppl (R Coll Pathol) 1974;8:156-63.

5. Nabarette H, Oziel D, Urbero B, et al. [Use of a directory of specialized services and guidance in the healthcare system: the example of the Orphanet database for rare diseases]. Rev Epidemiol Sante Publique 2006;54:41-53.

6. Caputo A. Exploring quality of life in Italian patients with rare disease: a computer-aided content analysis of illness stories. Psychol Health Med 2014;19: 211-21.

7. Remuzzi G, Garattini S. Rare diseases: what's next? Lancet 2008;371:1978-9.

8. Garrino L, Picco E, Finiguerra I, et al. Living with and treating rare diseases: experiences of patients and professional health care providers. Qual Health Res 2015;25:636-51.

9. EURORDIS. The voice of 12,000 patients. Experiences and expectations of rare disease patients on diagnosis and 
care in Europe: A report based on the EurordisCare2 and EurordisCare3 Survey; 2009. Available from: https:// www.eurordis.org/publication/voice12000-patients

10. Pierucci P, Lenato GM, Suppressa P, et al. A long diagnostic delay in patients with Hereditary Hemorrhagic Telangiectasia: a questionnaire-based retrospective study. Orphanet J Rare Dis 2012;7:33.

11. Zurynski Y, Deverell M, Dalkeith T, et al. Australian children living with rare diseases: experiences of diagnosis and perceived consequences of diagnostic delays. Orphanet J Rare Dis 2017;12:68.

12. Bloss S, Klemann C, Rother AK, et al. Diagnostic needs for rare diseases and shared prediagnostic phenomena: Results of a German-wide expert Delphi survey. PLoS One. 2017;12:e0172532.

13. Pasculli G, Resta F, Guastamacchia E, et al. Health-related quality of life in a rare disease: hereditary hemorrhagic telangiectasia (HHT) or Rendu-Osler-Weber disease. Qual Life Res 2004;13:1715-23.

14. Mazzucato M, Visona Dalla Pozza L, Minichiello C, et al. The epidemiology of transition into adulthood of rare diseases patients: Results from a population-based registry. Int $\mathrm{J}$ Environ Res Public Health 2018;15.

15. Sabbà C. Crescita esponenziale della fragilità dell'anziano in presenza di malattie rare. Giorn Gerontol 2014;62: 251-2.

16. Mazzucato M, Visona Dalla Pozza L, Manea S, et al. A population-based registry as a source of health indicators for rare diseases: the ten-year experience of the Veneto Region's rare diseases registry. Orphanet J Rare Dis 2014;9:37.

17. Lenato GM, Lastella P, Di Giacomo $\mathrm{MC}$, et al. DHPLC-based mutation analysis of ENG and ALK-1 genes in HHT Italian population. Hum Mutat 2006;27:213-4.

18. Sabba C, Pasculli G, Lenato GM, et al. Hereditary hemorrhagic telangiectasia: clinical features in ENG and ALK1 mutation carriers. J Thromb Haemost 2007;5:1149-57.

19. Sabba C, Pompili M. Review article: the hepatic manifestations of hereditary haemorrhagic telangiectasia. Aliment Pharmacol Ther 2008;28:523-33.

20. Sabba C, Pasculli G, Suppressa P, et al. Life expectancy in patients with hereditary haemorrhagic telangiectasia. QJM 2006;99:327-34.

21. de Gussem EM, Edwards CP, Hosman $\mathrm{AE}$, et al. Life expextancy of parents with hereditary hemorrhagic telangiectasia. Orphanet J Rare Dis 2016;11:46.

22. Mannucci PM, Iacobelli M. Progress in the contemporary management of hemophilia: The new issue of patient aging. Eur J Intern Med 2017;43:16-21.

23. Plug I, Van Der Bom JG, Peters M, et al. Mortality and causes of death in patients with hemophilia, 1992-2001: a prospective cohort study. J Thromb Haemost 2006;4:510-6.

24. Vaeth S, Vaeth M, Andersen H, et al. Charcot-Marie-Tooth disease in Denmark: a nationwide register-based study of mortality, prevalence and incidence. BMJ Open 2017;7:e018048.

25. Clegg A, Young J, Iliffe S, et al. Frailty in elderly people. Lancet 2013;381:752-62.

26. Sabbà C, Lenato GM, Suppressa P. Malattie "rare" in tarda età. In: Antonelli Incalzi R, Cesari M, Ungar A, Pedone C, Volpato S, Leosco D. Manuale di Geriatria. Milano, EDRA S.p.a. editions; 2019. pp 495-507

27. Shovlin CL, Millar CM, Droege F, Kjeldsen A, Manfredi G, Suppressa P, Ugolini S, Coote N, Fialla AD, Geisthoff U, Lenato GM, Mager HJ, Pagella F, Post MC, Sabba C, Sure U, Torring PM, Dupuis-Girod S, Buscarini E, Vascern HHT. Safety of direct oral anticoagulants in patients with hereditary hemorrhagic telangiectasia. Orphanet J Rare Dis. 2019;14(1):210. doi: 10.1186/s13023-019-1179-1. 